Thus

( $n$ even) $\underset{m=0}{\sum_{m}^{58}} y_{m} \cos 2 \pi \frac{n m}{60}=\sum_{m=0}^{15} d_{m} \cos 2 \pi \frac{n m}{60}$

( $n$ odd) $\underset{m=0}{\sum^{59}} y_{m} \cos 2 \pi \frac{n m}{60}=\sum_{m=0}^{14} e_{m} \cos 2 \pi \frac{n m}{60}$

( $n$ even) $\underset{m=0}{\sum^{59}} y_{m} \sin 2 \pi \frac{n m}{60}=\sum_{m=1}^{14} f_{m} \sin 2 \pi \frac{n m}{60}$

( $n$ odd) $\sum_{m=0}^{59} y_{m} \sin 2 \pi \frac{n m}{6 \jmath}=\sum_{m=1}^{\sum^{15}} g_{m} \sin 2 \pi \frac{n m}{60}$

The procedure is now as follows. Select from the box of strips the fourteen, fifteen or sixteen strips required for one group of summations. Separate these into two sets according as the value given on the strip for $n=15$ is zero or non-zero and arrange the two sets separately for addition of the columns of constant $n$. If an n-even group is being worked, total the columns for $n=0,2,4 \ldots$ in both sets; if an $n$-odd group is being worked, total the columns for $n=1,3,5 .$. in both sets. The totals for the two sets must be added according to columns to give the summations up to the fifteenth order of $n$. The totals for the two sets must be subtracted to give the higher orders of $n$ in descending order, the antisymmetric set being subtracted from the symmetric set.

The following simple checks are applicable (subsidiary checking is desirable for amplitude calculations and strip selection):

$$
\begin{aligned}
& \sum_{n=0}^{30} A_{n}=y_{0} ; \\
& \underset{\substack{n=1 \\
\sum^{30}}}{n=0}(-1)^{n / 2} A_{n}+\sum_{\substack{n=1 \\
n \text { even }}}^{\sum^{29}}(-1)^{(n-1) / 2} B_{n}=y_{15} .
\end{aligned}
$$

The whole process of computing and checking can be carried through for twenty-nine harmonics in less than two hours. The method has proved satisfactory in regular use and has been found less tiring than the process of curve tracing necessary in machine analysis. Except for convenience in checking, no more harmonics need be determined than are actually required. The procedure can be quickly learnt, errors are not liable to occur, and the calculations, including checking, can be performed almost entirely on an adding machine.

\section{Royal Circus,} Edinburgh.

1 Proc. Phy8. Soc., 48, 772 (1936). NATure, 137, 825 (1936).

${ }^{2}$ Whittaker and Robinson, "Calculus of Observations", pp. 264 et seq.

MIss Ross has done a useful service in directing attention to the value of the Beevers-Lipson strips for the process of Fourier analysis. These strips were originally designed for Fourier synthesis in crystal. structure determination, and for this purpose printed copies have been supplied to some forty crystalstructure laboratories (a few copies of the uncut tables are still available from this address). It seems likely, however, that the application to Fourier analysis is of rather wider interest. Prof. Max Born suggested this use of the strips to us some years ago, but it was only after inquiries by other workers, and finally by hearing of the successful work of Miss
Ross, that we have come to realize that the strips provide by far the most convenient practical method of Fourier analysis available in an ordinary laboratory.

The Fourier machine which we have recently described $^{1}$ will perform the same work as the strips, and would therefore carry out the process described by Miss Ross. It would enable the user to go from the numerical values of the $y_{m}$ to the twenty-nine Fourier coefficients in a time of less than eight minutes. Even faster working would be permitted by a minor extension of the machine so as to attain a wave-number of 30 instead of 24 as at present, and a machine working with similar components but with thirty-one counters and going up to a wave-number of 60 would enable the operator to complete the whole process in about three minutes. There may be applications of Fourier analysis where this extreme speed is desirable. Checking could be readily carried out by re-synthesis of the function.

The process can be done, of course, on a commercial scale by the use of the Hollerith series of tabulating machines, but these are considerably slower than the Beevers machine, although they provide a flexible and reliable method.

\section{Macewan.}

C. A. Beevers.

Dewar Crystallographic Laboratory, Chemistry Department,

King's Buildings,

Edinburgh, 9 . July 27.

${ }^{1}$ Beevers, Proc. Phys. Soc., 51, 660 (1939). Macewan and Beevers, J. Sci. Instr.. 19, 150 (1942).

\section{Temperature Gradients in Gaseous Explosions}

A NEw method of determining flame temperatures during explosions in a closed vessel has been evolved. Briefly, the method consists of recording photographically flame travel and simultaneously, by means of a very sensitive diaphragm type of pressure indicator, the pressure rise in a large spherical explosion vessel $17 \cdot 45 \mathrm{in}$. in diameter with central

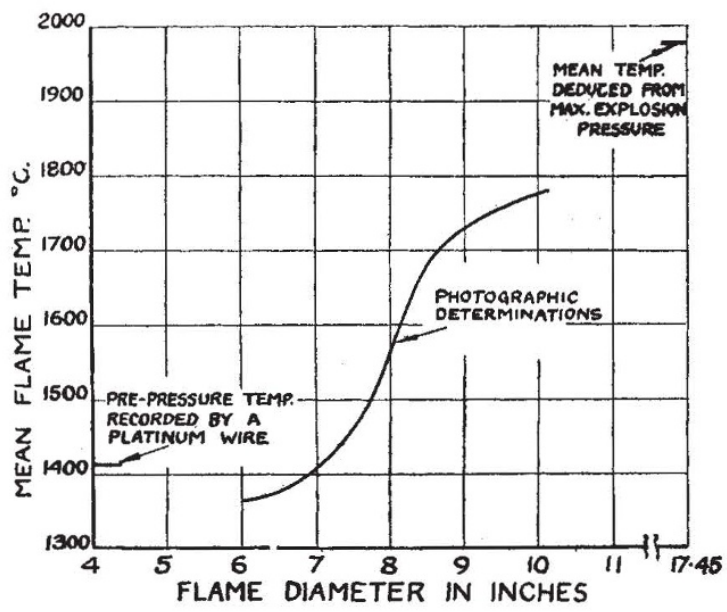

